\title{
Length of Stay: An Inappropriate Readout of the Success of Enhanced Recovery Programs
}

\author{
J. M. C. Maessen - C. H. C. Dejong • \\ A. G. H. Kessels - M. F. von Meyenfeldt • \\ on behalf of the Enhanced Recovery After Surgery (ERAS) Group
}

Published online: 29 January 2008

(C) The Author(s) 2008

\begin{abstract}
Background Enhanced recovery after surgery (ERAS) programs are designed to reduce hospital length of stay by shortening the postoperative recovery period. The intended effect of an accelerated recovery on the length of stay may be frustrated by a delayed discharge. This study was designed to assess the influence of an ERAS program on the proportion, appropriateness, and extent of delay in discharge.

Methods Patients who enrolled in the ERAS program $(\mathrm{n}=121)$ between 2003 and 2006 were compared with 52 patients who were managed traditionally in 2001.

Results Ninety percent of the pre-ERAS patients and $87 \%$ of the ERAS patients were not discharged on the day that discharge criteria were fulfilled. The additional stay of 59\% of the pre-ERAS patients and 69\% of the ERAS patients was inappropriate. Wound care (15\% in the pre-ERAS and $3 \%$ of the ERAS group) and observation of any symptoms pointing to an anastomotic leakage (10\% in both groups) were the most important reasons for a medical appropriate
\end{abstract}

J. M. C. Maessen $(\bowtie)$ · C. H. C. Dejong · M. F. von Meyenfeldt Department of Surgery, University Hospital Maastricht, PO Box 5800, 6202 AZ, Maastricht, the Netherlands e-mail: jmae@pmdi.azm.nl

\section{A. G. H. Kessels}

Department of Clinical Epidemiology \& Medical Technology Assessment, University Hospital Maastricht, Maastricht, the Netherlands

C. H. C. Dejong · M. F. von Meyenfeldt

Nutrim, Maastricht University, Maastricht, the Netherlands

on behalf of the Enhanced Recovery

After Surgery (ERAS) Group

Tromso, Norway delay of discharge. The extent of delay in discharge decreased significantly from a median of two days in the pre-ERAS group to a median of 1 day in the ERAS group $(p=0.004)$.

Conclusions Reductions in length of stay up to a median of 2 days after start of an enhanced recovery program may relate to changes in organization of care and not to a shorter recovery period. Recovery statistics should replace or at least be added to the length of stay as outcome of enhanced recovery programs.

\section{Introduction}

Because hospital services are the most expensive component of health care systems, hospitals are under increasing pressure to enhance the efficiency of hospital care. Length of stay for inpatient care is quoted as an important index of efficiency [1], and several changes in health care have been introduced during the past decades to limit the length of hospitalization.

An important development is the increased range of health care interventions offered outside the hospital or at home. External family accommodations near the hospital and home care services, such as visiting nurses for wound and stoma care, are now available to expedite the recovery of patients who no longer require the acute hospital setting.

In a parallel development, discharge planning programs, [2-4], discharge planning conferences [5] and even discharge professionals $[6,7]$ are introduced to facilitate the transmission of patients to the home or to other care services outside the hospital.

These measures are designed to reduce medically unnecessary hospital stays, notably the lower intensity days 
at the end of the hospital stay, during which the patient could have been discharged on clinical grounds but was not.

Protocols, clinical guidelines, and critical care pathways have been introduced in the belief that they have the potential to maintain the appropriateness of care offered while regulating inefficient hospital use [8-11].

In recent times, standardized perioperative care programs, with a more focused perioperative treatment plan designed to accelerate recovery, are in increasing in interest. Kehlet and colleagues [12, 13] demonstrated a dramatic reduction in hospital stay after abdominal surgery, by combining a series of interventions in perioperative care to shorten recovery time [14] with a number of actions to reduce unnecessary hospital stay. Compared with traditional care, there seems to be no difference in morbidity and mortality [15].

In 2000 the ERAS (Enhanced Recovery After Surgery) group was established, as a collaboration of five university or specialized departments of surgery. The ERAS group developed an evidence based perioperative care protocol for patients undergoing colorectal resection [16] and introduced this protocol in daily practice. To deal with the growing concern of caregivers regarding adverse outcomes in patients who were discharged early, the ERAS group defined criteria to be fulfilled before discharge [16].

A first evaluation of the program showed that almost $70 \%$ of the patients were not going home on the day that discharge criteria were fulfilled and that any delay in discharge was strongly associated with a prolonged hospital length of stay [17].

In the current study, this gap between the moment a patient could go home theoretically, based on predefined discharge criteria, and the moment of actual discharge was evaluated.

The objectives were to assess the influence of the enhanced recovery after surgery program on the proportion, appropriateness, and extent of delay in discharge.

\section{Materials and methods}

The ERAS program for patients undergoing elective colonic resections above the peritoneal reflection without formation of a stoma was instituted in the beginning of 2002. With a focus on stress reduction and promotion of return to function, the goal of the ERAS program is quicker recovery from major abdominal surgery with a reduced hospital stay as a consequence. Preoperative preparation and medication, minimal invasive surgery, optimal pain relief, early nutrition, and enforced mobilization are key elements of an enhanced recovery approach.

An analysis of perioperative care before initiation of the program showed that the principles of the ERAS program were not adopted in patients who underwent a colonic resection in 2001 and that the perioperative routines did not differ from the traditional routines as published by $\mathrm{K}$. Lassen [18, 19].

The traditionally managed patients in 2001 (the preERAS group) were compared with the patients who were managed according to the ERAS program between 2003 and 2006 (the ERAS group). The study included consecutive patients undergoing elective colorectal resection above the peritoneal reflection, without formation of a stoma.

Patients treated in 2002 were excluded to guarantee the study group to be managed according to the fully implemented new program to exclude transition effects and to consist of consecutively treated patients. Before start of the study, ethical approval for collection of the data was obtained.

Data for the ERAS group were assessed prospectively. Data for the pre-ERAS group were obtained retrospectively by analysis of medical and nursing charts.

In the current study, primary outcome was delay in discharge. Discharge criteria were defined as tolerance of food, good pain control on oral analgesics, defecation, and independence in activities of daily living (ADL) to preoperative care level.

The first day postoperatively that a patient fulfilled all four discharge criteria was considered to be the day that the patient was recovered and ready for discharge. Delay in discharge was defined as any difference between the moment the patient was ready for discharge and the actual discharge.

Recovery data were recorded for 30 days after surgery. Seven percent of the patients in the pre-ERAS group and $8 \%$ of the patients in the ERAS group were not ready for discharge within 30 days after surgery (died, still in hospital, or discharged to a higher care level). These cases were omitted from analysis.

The Dutch model of the Appropriateness Evaluation Protocol (DAEP) was used to assess the appropriateness of hospital stay in case of a delay in discharge. Appropriate hospital stay was defined as: "Hospital stay, requiring continuous and active medical, nursing or paramedical treatment, which under existing legislation cannot be provided through extramural care, day care, or outpatient care" [20].

The DAEP is a modification of the U.S. Appropriateness Evaluation Protocol and was proven to be a valid, reliable, and user-friendly instrument for evaluating the appropriateness of hospital stay [21]. The DAEP consists of 19 criteria for appropriate hospital stay. 
Statistics

Data are given as mean (SD) or median (range). Differences in demographic and clinical variables were analyzed by using Mann-Whitney test for ordinal data and $\chi^{2}$ test for categorical data. $P$ values $<0.05$ were considered to indicate statistical significance. Data analyses were performed with SPSS ${ }^{\circledR} 12.1$ for Windows XP (SPSS, Inc., Chicago, IL).

\section{Results}

Patient characteristics are presented in Table 1 and demonstrate that the two study groups were comparable with respect to demographic variables and surgical procedures. Table 2 shows that there were no significant differences in the proportion and the appropriateness of the delay in discharge between the two study groups.

Ninety percent of the pre-ERAS patients and 87 percent of the ERAS patients were not going home on the day that discharge criteria were met. The prolonged hospital stay of $59 \%$ of the pre-ERAS patients and 69\% of the ERAS patients was labeled as inappropriate, according to the DAEP. For $31 \%$ of the pre-ERAS group and $18 \%$ of the ERAS group, the prolonged stay was rated as medically necessary, with as main reason wound care $(15 \%$ of the patients of the pre-ERAS group and $3 \%$ of the ERAS group) and observation of any symptoms pointing to an anastomotic leakage (10\% in both the pre-ERAS and ERAS group).

The extent of the delay was significantly reduced in the ERAS group. The additional stay after full recovery, according to the predefined discharge criteria, was a

Table 1 Comparison of patient characteristics of traditionally managed patients in 2001 (the pre-ERAS group) and patients managed according to the enhanced recovery after surgery program between 2003-2006 (ERAS group)

\begin{tabular}{lll}
\hline & $\begin{array}{l}\text { Pre-ERAS } \\
(\mathrm{n}=52)\end{array}$ & $\begin{array}{l}\text { ERAS } \\
(\mathrm{n}=121)^{\mathrm{a}}\end{array}$ \\
\hline Age mean (SD) & $64(11.8)$ & $66(12.3)$ \\
Male/female ratio (\%) & $42 / 58$ & $55 / 45$ \\
ASA classification III/ IV & 10 & 20 \\
$\quad(\%)$ & & \\
Colectomy (\%) & & \\
$\quad$ Right-sided & 44 & 45 \\
$\quad$ Left-sided & 56 & 55 \\
Complex resection (\%) & 12 & 74 \\
Malignancy (\%) & 75 & $31(6.9)$ \\
P-Possum mean (SD) & $30(6.2)$ & \\
\hline
\end{tabular}

${ }^{a}$ No significant differences median of 2 (range, 0-17) days in the pre-ERAS group versus a median of 1 (range, 0-9) day in the ERAS group $(p=0.004)$. The main cause of the shorter delay in discharge of ERAS patients was the reduced incidence of wound infections during hospital stay.

\section{Discussion}

The main rationale for implementing enhanced recovery programs in surgical practice is that an improved and shorter (enhanced) recovery period would reduce a patient's need to stay in the hospital and thus reduce the length of hospitalization [16] —a simple concept, but particularly revolutionary [22] because it refers exclusively to a reduction in length of stay by influencing the number of the high intensity first days after surgery.

As the present study demonstrates, the intended effect of a shorter recovery period on length of stay is frustrated in part by an additional stay in hospital after the patient has fully recovered according to predefined discharge criteria. The current study shows that there was no significant difference in the proportion of patients with a delay in discharge, before and after start of the ERAS program. Approximately $90 \%$ of the patients were not discharged on the day that functional recovery was achieved.

A validated appropriateness instrument, the Dutch Appropriateness Evaluation protocol (DAEP), was used to judge whether the stay after recovery criteria were fulfilled was medically necessary. According to the DAEP, the prolonged stay of $60 \%$ of the pre-ERAS patients and $70 \%$ of the ERAS patients was deemed inappropriate. The discharge scoring system defined adequately the readiness to go home of these patients, who had no medical reasons to stay in hospital after discharge criteria were fulfilled.

In $30 \%$ of the pre-ERAS patients and $20 \%$ of the ERAS patients, the prolonged stay after recovery was rated as medically necessary. According to the discharge criteria, these patients were ready to go home, but the DAEP judged that these patients had medical reasons for a prolonged stay, with as main medical reasons wound care and observation of any symptoms pointing to an anastomotic leakage.

The question is whether wound care and observation of symptoms require a hospital setting to be managed adequately. According to the recent changes in the perception of the role of an acute hospital bed [22-24], wound care and observation of symptoms are both debatable reasons for staying in an acute hospital bed.

The lower incidence of prolonged stay because of wound care in the ERAS group (3\% vs. 15\%) illustrates that wound care can be considered as an inappropriate 
Table 2 Proportion, appropriateness, and extent of delay of discharge before and after start of the ERAS program

\begin{tabular}{lcr}
\hline & $\begin{array}{l}\text { Pre-ERAS } \\
(\mathrm{n}=52)\end{array}$ & \multicolumn{1}{l}{$\begin{array}{l}\text { ERAS } \\
(\mathrm{n}=121)\end{array}$} \\
\hline $\begin{array}{l}\text { Delay in discharge } \\
\quad \text { No }\end{array}$ & $5(10 \%)$ & $16(13 \%)$ \\
$\quad$ Yes & $47(90 \%)$ & $105(87 \%)$ \\
Medically inappropriate & $31(59 \%)$ & $83(69 \%)$ \\
$\begin{array}{l}\text { Medically appropriate } \\
\begin{array}{l}\text { Median extent of delay in discharge } \\
\text { (range) }\end{array}\end{array}$ & $2(0-17)$ & $1 *(0-9)$ \\
\hline
\end{tabular}

$* p=0.004$

reason for a prolonged stay. The incidence of wound infections was not influenced by the ERAS program; however, more wound infections occurred at home and were subsequently treated in the outpatient setting.

In both groups, $10 \%$ of the patients were not discharged because of fever, abdominal distention, diarrhea, or other symptoms that could point to an anastomotic leakage. These symptoms resolved within some days, and none of these patients actually developed an anastomotic leakage. It is part of our traditional thinking that patients should remain in the hospital after surgery to be observed for any complication that may occur; the main advantage is the immediate response to complications and the rapid access to repeat surgery. However, with repetitive phone calls after discharge or home care visits and a rapid access to the outpatient surgery department, observation of patients may become an inappropriate reason for offering an acute hospital bed. If both would be rated as inappropriate reasons for stay, only $5 \%$ of patients have a delay in discharge that could be categorized as truly medically appropriate.

Actually, the discrepancy between the DAEP and the discharge scoring system shows that besides the medical requirements for hospitalization expressed in the discharge criteria, length of stay is influenced by local perceptions and organization of the acute hospital bed. The length of the additional stay, beyond the "fit for discharge" point, was a median of 2 days in the traditionally managed group and a median of 1 day in the enhanced recovery group. This statistically significant and clinically important reduction in delay after the start of the ERAS program was mainly caused by the reduction in the incidence of wound infections during hospital stay, which was an unintended effect of the ERAS program.

This observation confirms that reductions in length of stay up to a median of 2 days are not necessarily related to the effect of the program on the recovery period. Instead, they may relate to changes in local nursing and doctor's policy, bringing the point of actual discharge closer to the fit for discharge moment.
Therefore, recovery criteria are superior to length of stay for the evaluation of the success of an enhanced recovery program. The first day that the patient is able to eat, has good pain control while taking oral analgesics, has had defecation, and is nursed back to ADL self-care must be the most important outcome parameter of enhanced recovery programs, because it defines most adequately the recovery of the patient and eliminates the influence of the organization of care.

Open Access This article is distributed under the terms of the Creative Commons Attribution Noncommercial License which permits any noncommercial use, distribution, and reproduction in any medium, provided the original author(s) and source are credited.

\section{References}

1. Health Do (2002) NHS perfomance indicators. London: Department of health

2. Shepperd S, Parkes J, McClaran J, Philips C (2006) Discharge planning from hospital to home (review). The Cochrane Library

3. Patterson CJ, Mulley GP (1999) The effectiveness of predischarge home assessment visits: a systematic review. Clin Rehabil 13:101-104

4. Hyde CJ, Robert IE, Sinclair AJ (2000) The effects of supporting discharge from hospital to home in older people. Age Ageing 29:271-279

5. Efraimsson E, Sandman PO, Hyden LC, Holritz Rasmussen B (2006) How to get one's voice heard: the problems of the discharge planning conference. J Adv Nurs 53:646-655

6. Armitage SK, Kavanagh KM (1996) The discharge liaison nurse at the interface of hospital and community nursing services. Int $\mathrm{J}$ Nurs Pract 2:215-221

7. Dukkers van Emden DM, Ros WJ, Berns MP (1999) Transition of care: an evaluation of the role of the discharge liaison nurse in The Netherlands. J Adv Nurs 30:1186-1194

8. Wollersheim H, Burgers J, Grol R (2005) Clinical guidelines to improve patient care. Neth J Med 63:188-192

9. Weingarten S, Riedinger MS, Sandhu M, Bowers C, Ellrodt AG, Nunn C, Hobson P, Greengold N (1998) Can practice guidelines safely reduce hospital length of stay? Results from a multicenter interventional study. Am J Med 105:33-40

10. Pearson SD, Goulart Fisher D, Lee TH (1995) Critical pathways as a strategy for improving care: problems and potential. Ann Intern Med 123:941-948

11. Cook DJ, Greengold NL, Ellrodt AG, Weingarten SR (1997) The relation between systematic reviews and practice guidelines. Ann Intern Med 127:210-216

12. Kehlet H, Mogensen T (1999) Hospital stay of 2 days after open sigmoidectomy with a multimodal rehabilitation programme. Br J Surg 86:227-230

13. Basse L, Hjort Jakobsen D, Billesbolle P, Werner M, Kehlet $\mathrm{H}$ (2000) A clinical pathway to accelerate recovery after colonic resection. Ann Surg 232:51-57

14. Kehlet H, Wilmore DW (2002) Multimodal strategies to improve surgical outcome. Am J Surg 183:630-641

15. Nygren J, Hausel J, Kehlet H, Revhaug A, Lassen K, Dejong C, Andersen J, von Meyenfeldt M, Ljungqvist O, Fearon KC (2005) A comparison in five European Centres of case mix, clinical management and outcomes following either conventional or fast-track perioperative care in colorectal surgery. Clin Nutr 24: $455-461$ 
16. Fearon KC, Ljungqvist O, Von Meyenfeldt M, Revhaug A, Dejong $\mathrm{CH}$, Lassen K, Nygren J, Hausel J, Soop M, Andersen J, Kehlet H (2005) Enhanced recovery after surgery: a consensus review of clinical care for patients undergoing colonic resection. Clin Nutr 24:466-477

17. Maessen J, Dejong CHC, Hausel J, Nygren J, Lassen K, Andersen J, Kessels AGH, Revhaug A, Kehlet H, Ljungqvist O, Fearon $\mathrm{KCH}$, Meyenfeldt MFV (2007) A protocol is not enough to implement an enhanced recovery programm for colorectal resection. Br J Surg 94(2):224-231

18. Lassen K, Hannemann P, Ljungqvist O, Fearon K, Dejong $\mathrm{CH}$, von Meyenfeldt MF, Hausel J, Nygren J, Andersen J, Revhaug A (2005) Patterns in current perioperative practice: survey of colorectal surgeons in five northern European countries. BMJ 330:1420-1421

19. Lassen K, Dejong CH, Ljungqvist O, Fearon K, Andersen J, Hannemann $\mathrm{P}$, von Meyenfeldt MF, Hausel J, Nygren J,
Revhaug A (2005) Nutritional support and oral intake after gastric resection in five northern European countries. Dig Surg 22:346-352

20. Panis LJ, Verheggen FW, Pop P, Prins MH (2004) Appropriate length of extended day care. Int J Health Care Qual Assur Inc Leadersh Health Serv 17:81-86

21. Smeets PM, Verheggen FW, Pop P, Panis LJ, Carpay JJ (2000) Assessing the necessity of hospital stay by means of the appropriateness evaluation protocol: how strong is the evidence to proceed? Int J Qual Health Care 12:483-493

22. Clarke A, Rosen R (2001) Length of stay. How short should hospital care be? Eur J Public Health 11:166-170

23. Clarke A (2002) Length of in-hospital stay and its relationship to quality of care. Qual Saf Health Care 11:209-210

24. Schoetz DJ Jr, Bockler M, Rosenblatt MS, Malhotra S, Roberts PL, Murray JJ, Coller JA, Rusin LC (1997) "Ideal” length of stay after colectomy: whose ideal? Dis Colon Rectum 40:806-810 